\title{
THE GAMMA-RAY PROPERTIES OF UNIDENTIFIED EGRET SOURCES
}

\author{
Olaf Reimer \\ Laboratory for High Energy Astrophysics \\ NASA Goddard Space Flight Center \\ Greenbelt, $M D 20771$ \\ $U S A$ \\ olr@egret.gsfc.nasa.gov
}

Keywords: High energy gamma-ray sources, unidentified EGRET sources, population studies

\begin{abstract}
Although the majority of gamma-ray sources still remain unidentified, we have various kinds of information to characterize the observational properties of unidentified EGRET sources. Despite astronomical properties like locations of individual sources or the collective arrangement of the class as such, the nine years of CGRO observations provide the ability to investigate flux variability at different timescales, enable us to perform periodicity searches, determine gamma-ray source spectra between $30 \mathrm{MeV}$ and $10 \mathrm{GeV}$ and even investigate spectral variability. The basic observational properties of unidentified high-energy gamma-ray sources discovered by EGRET are reviewed. Various instrumental and observational peculiarities affecting the interpretation of the EGRET data are pointed out, also describing the way such biases might affect scientific conclusions drawn from the EGRET data.
\end{abstract}

\section{INTRODUCTION}

With NASAs Compton Gamma-Ray Observatory mission terminated in June 2000, the EGRET data base will remain a unique and extremely important source of scientific information. Although being an archival data base from now on, it has to be considered as state-of-the-art for several more years, because presently no other instrument covering the high-energy gamma-ray wavelengths is in orbit. Even when instruments like AGILE and GLAST will become operational, the EGRET data will be the reference for new observational results. CGROs coverage of a long time period between 1991 and 2000 will be used in determining the 
long-term behaviour of gamma-ray sources and very likely for subsequent archival research. To a lesser degree, this has been already demonstrated by comparing EGRET data with results from previous missions, in particular with COS-B. Not only were earlier reported source detections considered for positional comparison, in cases like Geminga archival data were analyzed in conjunction with the EGRET data for tracing its long term periodicity behavior. When going into the subject of discussing unidentified EGRET sources, COS-B source findings are still an interesting aspect of reference, since predictions from COS-B population studies could be supported or rejected using the EGRET data or to establish the long-term coverage of individual sources (i.e. 2CG $135+1$ or $2 \mathrm{CG} 075+0)$. Here, I will review how EGRET data were used to construct the source catalogs, flux histories and source spectra, and discuss the pecularities of the existing point-source catalogs, their positional accuracy and underlying systematics. The importance of understanding in which way such biases might affect scientific conclusions will be addressed. Also, different approaches to deal with variability are compared. Quantitatively, the flux determination needs to be related to EGRETs instrumental response in orbit over time and energy. The determination of photon spectra will be discribed and a view beyond simple single power-law fits needs to be given. In several cases with exceptional observational coverage, also spectral variabilty could be adressed. Having accumulated detailed knowledge of the spacial, temporal and spectral properties of individual unidentified EGRET sources, the quest for finding signatures in the collective could be challenged. Conclusions are drawn on the validity of assumptions, selections and cuts in population studies, mainly under aspects of known instrumental biases or pecularities with the gamma-ray point source catalogs and questioning barely justified speculations.

\section{EGRET SOURCE CATALOGS AND GAMMA-RAY SOURCE LOCATIONS}

Omitting all low level EGRET data products and therefore the complete process of event reconstuction and event quality classes, the Third EGRET catalog of high-energy gamma-ray sources (Hartman et al. 1999) has been constructed on the basis of individual viewing periods, meaningfully selected combinations of individual viewing periods (i.e. subsequent short observations or sequences with nearly identical pointings) and annual superpositions of all observations synchronized with CGRO observation cycles. For each of those selections appropriate count, exposure and intensity maps have been constructed, usually by applying 
a standard $<30^{\circ}$ field-of-view cut. These maps have been analysed by means of a maximum-likelihood procedure (Mattox et al. 1996). Although fluxes are consistently given for $\mathrm{E}>100 \mathrm{MeV}$, similar analyses have been performed for the energy intervals $300-1000 \mathrm{MeV}$ and above $1000 \mathrm{MeV}$. The different likelihood test statistics (TS) maps were compared and, as long as $(\mathrm{TS})^{1 / 2}>4$, the one which produced the smallest error contours was chosen to represent the actual source location. Figure 1 shows the EGRET all-sky TS map, the result of the maximum-likelihood analysis procedure carried out from all viewing periods between April 1991 and September 1995 at $>100 \mathrm{MeV}$. The in-

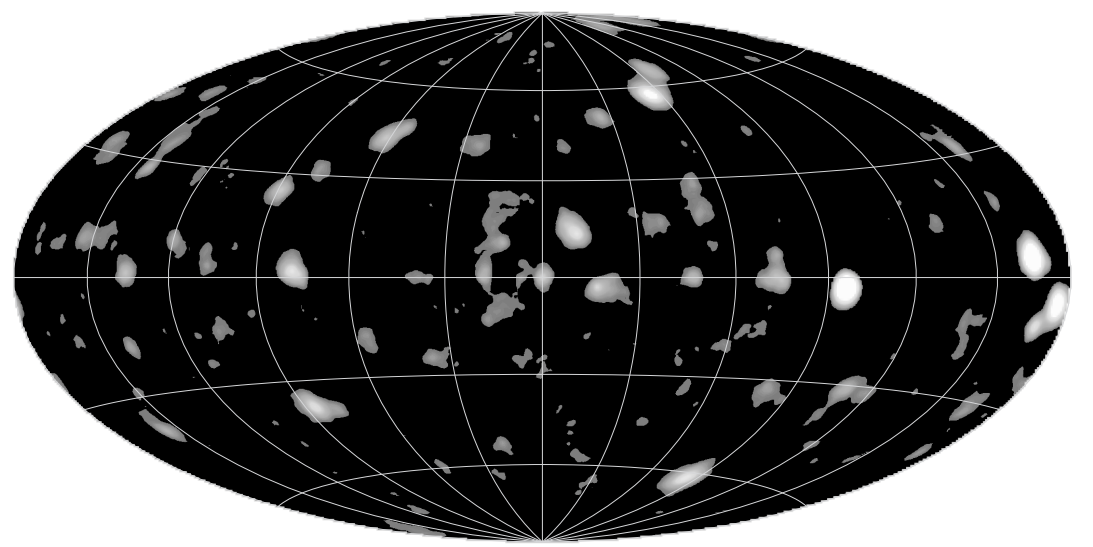

Figure 1 Test statistics (to say detection significance) map as result of the maximumlikelihood analysis of the EGRET data from cycle 1 to 4 observations

tense and highly structured diffuse emission along the Galactic planes makes the determination of sources in the Galactic plane more subject to systematic uncertainties than those at higher latitudes. Therefore, the detection criterion for a gamma-ray source is also different for its location: In at least one of the derived maps $(\mathrm{TS})^{1 / 2}>4$ for sources at $|b|>10^{\circ}$ or $(\mathrm{TS})^{1 / 2}>5$ at $|b|<10^{\circ}$ must be fulfilled. This peculiar step in the acceptance criterion is somewhat arbitrary, however translates directly into a bias taken care of in source population studies. Additionally, the acceptance criterion could be fulfilled in any of the derived maps, resulting in detections on the basis of very uneven exposure times. One-time flaring sources will be included as well as sources which build up the detection significance in a purely statistical way originating from more and more observations, i.e. exposure. The resulting point source catalog (Fig.2) therefore represents a rather uneven sample for population study applications.

Also, the underlying diffuse emission model (Hunter et al. 1997) is 
known to be imperfect on smaller scales. The likelihood source detection algorithm will translate such inaccuracies of the diffuse model into the detection significance and, very likely, into acceptance issues for sources near the catalog thresholds. The procedure of independently scaling of the nominal values of the diffuse emission model within the radiusof-analysis (usually $15^{\circ}$ ) in order to account for small-scale structures might not be in each case the most accurate one in order to discriminate point-like excesses against features in the diffuse emission. In confused regions with sources of significantly overlapping point spread functions (PSF), the order in which sources are optimized becomes important, too. These systematics are generally not easy to quantify, but obviously need consideration in 3EG catalog-based population studies. Figure 2 shows the high-energy gamma-ray sources fulfilling the $3 \mathrm{EG}$ catalog acceptance criteria. The size of the symbols represents the maximum intensity seen for this source.

With the pecularities of the construction of the Third EGRET catalog

\section{Third EGRET Catalog}

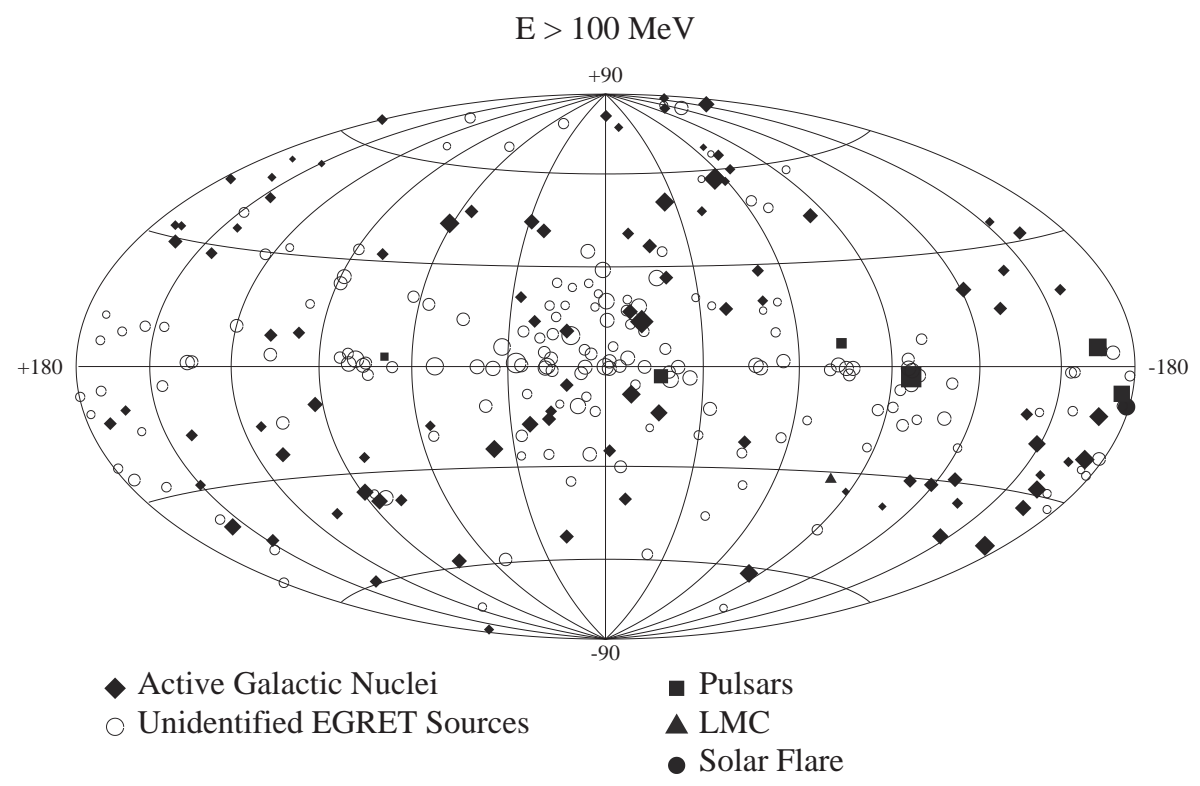

Figure 2 Gamma-ray point sources in the Third EGRET catalog

in mind, nevertheless the longitudinal and latitudinal characteristics of the sample can be sketched. Fig. 3 shows the complete 3 EG catalog sample (outlined) and a selection made of sources fulfilling a common 
$(\mathrm{TS})^{1 / 2}>5$ acceptance criterion on the basis of the summed map from CGRO observation cycles 1 to 4 only (shaded). As can easily be seen, the distribution of unidentified sources represents a distinct Galactic population, but also shows some spreading at mid-latitudes on top of a rather flat component present at all latitudes.

Additional compilations of high-energy sources have been made, i.e.
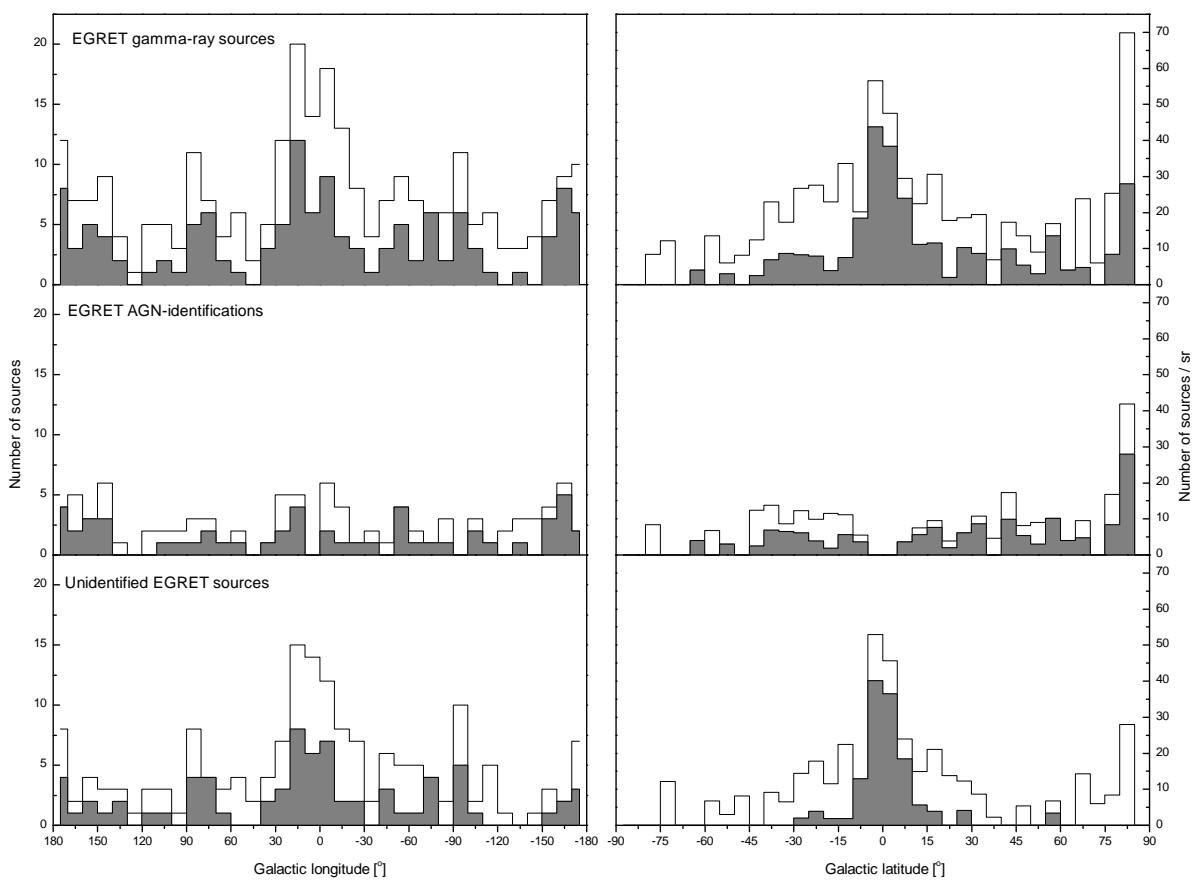

Figure 3 Longitudinal and latitudinal distributions of the 3 EG catalog sources. See text for details.

for $\mathrm{GeV}$ sources (Lamb \& Macomb 1997, Reimer et al. 1997). The obvious reason to compile a catalog of a higher energy threshold is the significantly reduced Galactic diffuse emission component (and therefore surpression of systematic uncertainties originating from it during source determination procedures) in conjunction with a narrower instrumental PSF. These advantages are offset by the reduced photon flux at higher energies and therefore a loss in photon statistics. However, in cases of bright sources, hard photon spectral indices or regions suffering from source confusion, the trade between limited statistics and better angular resolution often leads to significantly narrower error contours. Figure 4 compares the error contours of the unidentified high-latitude source 
3EG J2020+4014 ( $\gamma$ Cyg) at energies above $100 \mathrm{MeV}$ and above $1 \mathrm{GeV}$, respectively. A proper way to treat error contours in a mathematical-
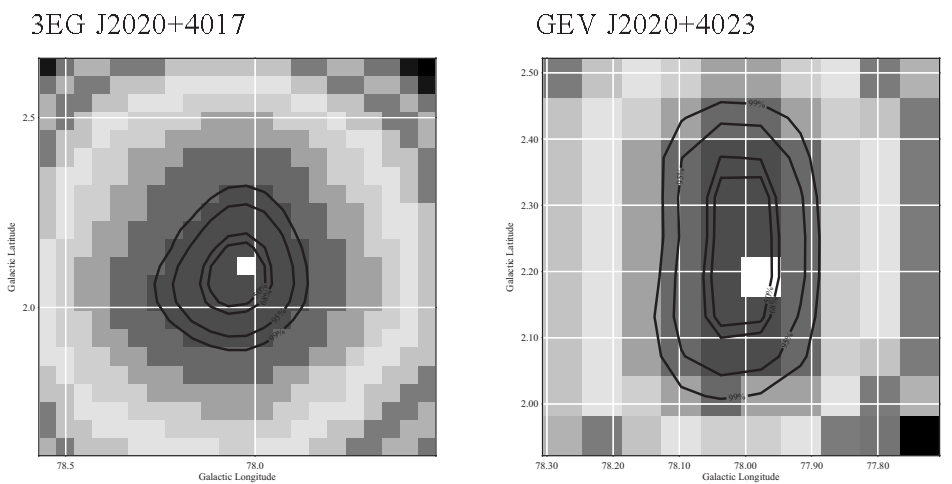

Figure 4 Gamma-ray emission from $\gamma$ Cygni at $\mathrm{E}>100 \mathrm{MeV}$ and $\mathrm{E}>1 \mathrm{GeV}$, respectively. Note the different scaling of the figures. The visible pixelation of $0.05^{\circ}$ by $0.05^{\circ}$ is the same in both images. The best position is similar, but the uncertainties are smaller at higher energies, dedicating the GeV-image for counterpart studies.

statistical way (i.e. for counterpart propability tests) has been found in elliptical fits to source contours. Narrower error contours to a gammaray source do not automatically imply a more exact point source location in the coordinates. Comparing the catalog source positions determined at different energety thresholds with precise coordinates of astronomical objects could only be performed if an identification has been established, i.e. from observations at other wavelengths. For the high-energy gammaray sources this can be accomplished using pulsars (PSRs) and active galactic nuclei (AGN). Figure 5 compares the gamma-ray source locations from the 3EG catalog (Hartman et al. 1999) and the GeV-catalog (Lamb \& Macomb 1997) with the radio positions of these objects, providing estimates of the precision with which EGRET typically determines gamma-ray point source coordinates.

\section{EGRET SOURCE DETECTIBILITY AND CONSEQUENCES}

The significance $\mathrm{s}$ for a detection of an isolated point source with EGRET $(\mathrm{E}>100 \mathrm{MeV})$ is adequately represented by

$$
s \propto f \sqrt{\frac{e}{b g}},
$$

where $\mathrm{f}$ is the flux, e the exposure and bg the intensity of the diffuse gamma-ray emission at the region of the source (Mattox et al. 1996). 

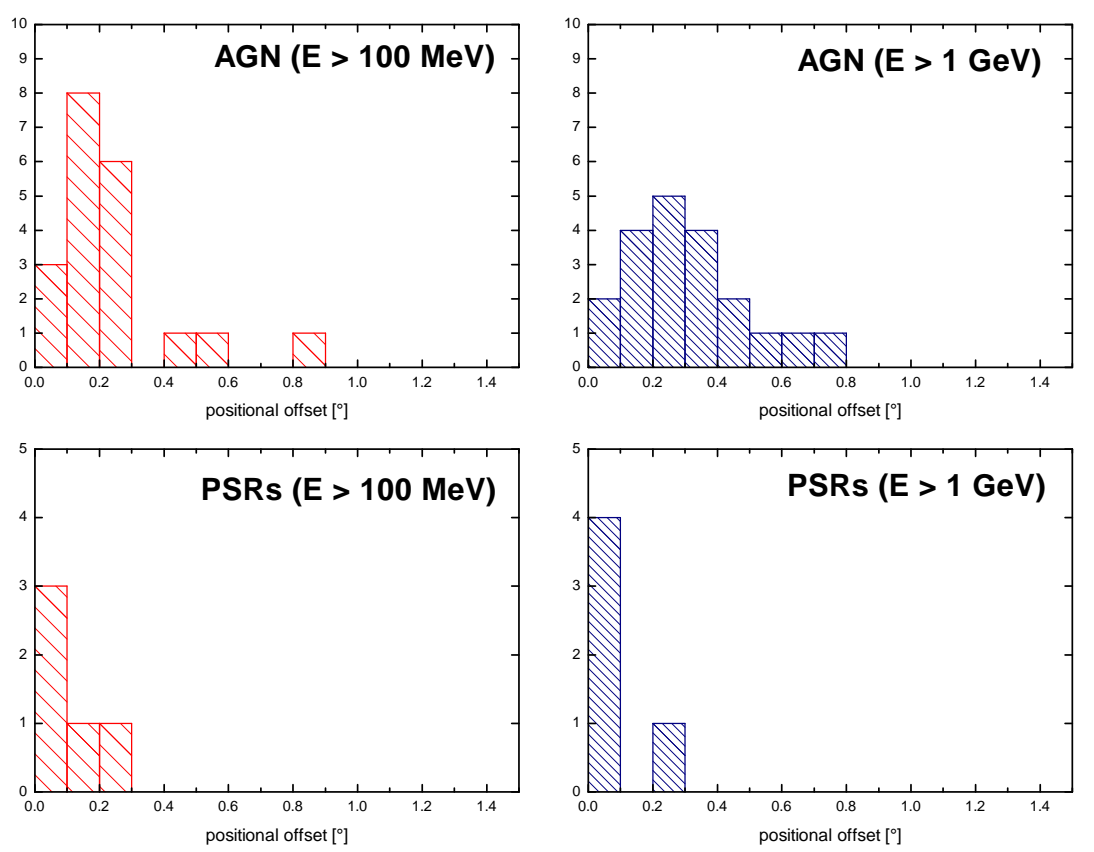

Figure 5 Source location offsets comparing the gamma-ray coordinates of identified objects with precise coordinates from optical or radio counterparts. The GeV positions are not significantly more precise than those determined for $\mathrm{E}>100 \mathrm{MeV}$.

In order to simulate a corresponding picture of the gamma-ray sky as given in the 3EG catalog, all three observables needs to be looked at in detail.

- Exposure: The observational history of the EGRET instrument is highly non-uniform and so is the exposure. EGRET pointings have a typical field-of-view of $40^{\circ}$, however for most applications a $30^{\circ}$ cut is recommended as applied, for instance, in the $3 \mathrm{EG}$ catalog. If population studies compare with the EGRET source catalog, the exposure needs to be determined for each individual source. As mentioned above, catalog sources are not consistently included on the basis of equal exposure time. Therefore, exposure time as well as the corresponding number of sources matching the detection criterion at any considered time interval should be looked at.

- Diffuse gamma-ray emission: The detectibilty for EGRET sources also depends on the diffuse gamma-ray background in the source region. To compensate for these nonuniformities, several ways are applicable. The diffuse gamma-ray emission model constructed 
from HI and CO distributions (Hunter et al. 1997) is available in the standard EGRET energy intervals. Alternatively, a pointsource-removed intensity map could be used as has been done by Strong, Moskalenko \& Reimer 2000. Although both approaches are different in the scientific content, they provide a good measure of the characteristics of the diffuse gamma-ray emission for the purpose of use in population studies.

- Flux: Monte-carlo based population simulations are required to reproduce the actually observed $\log \mathrm{N}-\log \mathrm{S}$ distribution of gammaray sources, globally as well as locally. As noticed by Gehrels et al. 2000, the $\log \mathrm{N}-\log \mathrm{S}$ distribution for unidentified sources close to the Galactic plane differs from the one obtained at high Galactic latitudes, although partly as a result of the nonuniform detectibility function of EGRET itself. Nevertheless, the $\log \mathrm{N}-\log \mathrm{S}$ of identified gamma-ray sources is distinctly different, i.e. for AGN (Özel \& Thompson 1996). Estimates of the fraction of unresolved point sources will come to play when concluding on the basis of simulations involving large numbers of sources as expected in the GLAST-era.

A rather simplified approch to account for the EGRET detectibilty function can be made by determining upper limits for a grid on the sky. These upper limits needs to be determined at comparable statistical significance. An example is given in the 3EG catalog, using the summed exposures of CGRO cycles 1, 2, 3, and 4. Note that the catalog acceptance criterion and $95 \%$ confidence upper limits leave room for excesses in the test statistics inbetween, which needs to be accounted for in simulations. The full composition of the $3 \mathrm{EG}$ catalog might be investigated by following this scheme through each of the individual viewing periods in order to account for transient sources. Lastly, upper limits near bright catalog sources are expected to be underestimated due to the width of the EGRET point spread function.

Figure 6 shows a detectibility map for equally bright sources, determined for EGRET observations from CGRO cycles 1, 2, 3, and 4 (E> 100 $\mathrm{MeV})$. Note the features close to the Galactic plane, where the low detectibility directly in the plane increasingly becomes compensated from the exposure. However, the highly nonuniform character of EGRETs detectibility function is easy to recognize. On a $0.5^{\circ} \times 0.5^{\circ}$ grid, the value of the detectibilty function for sources of comparable flux differs by more than a factor of ten! 


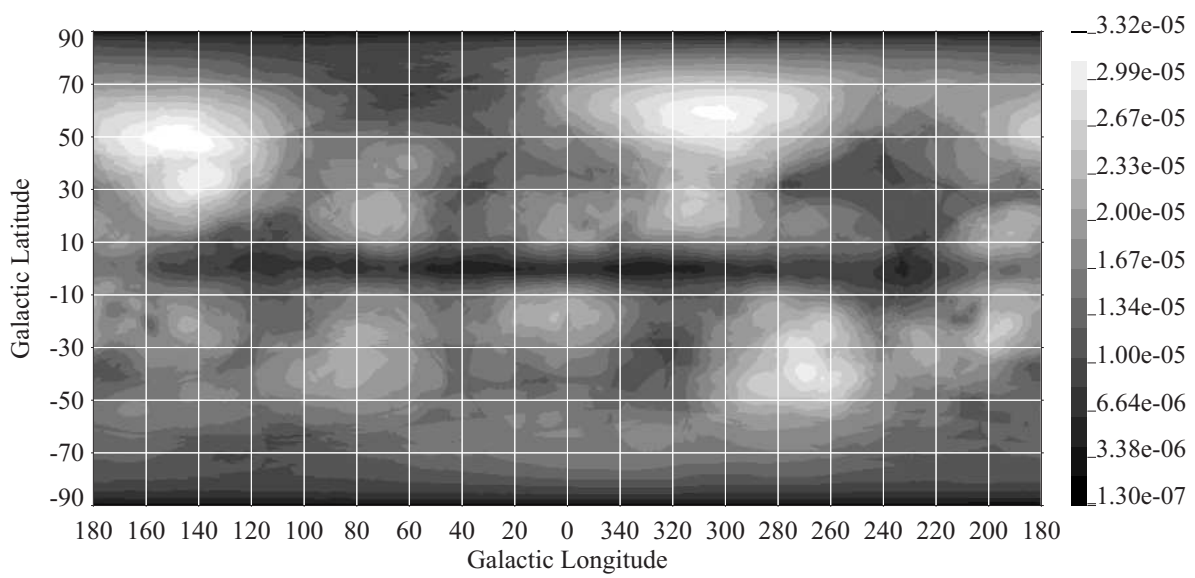

Figure 6 Two-dimensional detectibility function for gamma-ray sources above 100 $\mathrm{MeV}$ and comparable flux. This map corresponds only to the analysis of the summed EGRET cycle 1 to 4 observations, as listet as P1234 in the 3 EG catalog (units of $\mathrm{cm}^{2}$ s).

\section{VARIABILITY OF GAMMA-RAY SOURCES}

Gamma-ray source variability is even more difficult to quantify than test statistics excesses, nonuniform detection thresholds and absolute coordinates for gamma-ray sources. At first glance, one needs to look at the spark chamber efficiency of the EGRET instrument. As discribed in Esposito et al. 1999, the spark chamber efficiency is strongly time-dependent. For any meaningful determination of gamma-ray source fluxes, the response has to be normalized. Scale-factors have been constructed for a given energy and time interval, primarily by comparing the level of the ever present diffuse emission component. Neither the determination of the inital response nor the scaling to a nominal value is perfect. Although we think that the response of the spark chamber should underly a rather smooth degradation between major events impacting its performance (gas refills), some individual viewing periods appear to be slightly off the generally smooth normalization scheme. However, the individual normalization factors are accessible for each observation period and the individual case could be well investigated beyond the flux value listed in a source catalog. Figure 7 gives an impression of the spark chamber efficiency versus time, before and after the normalization has been applied. 


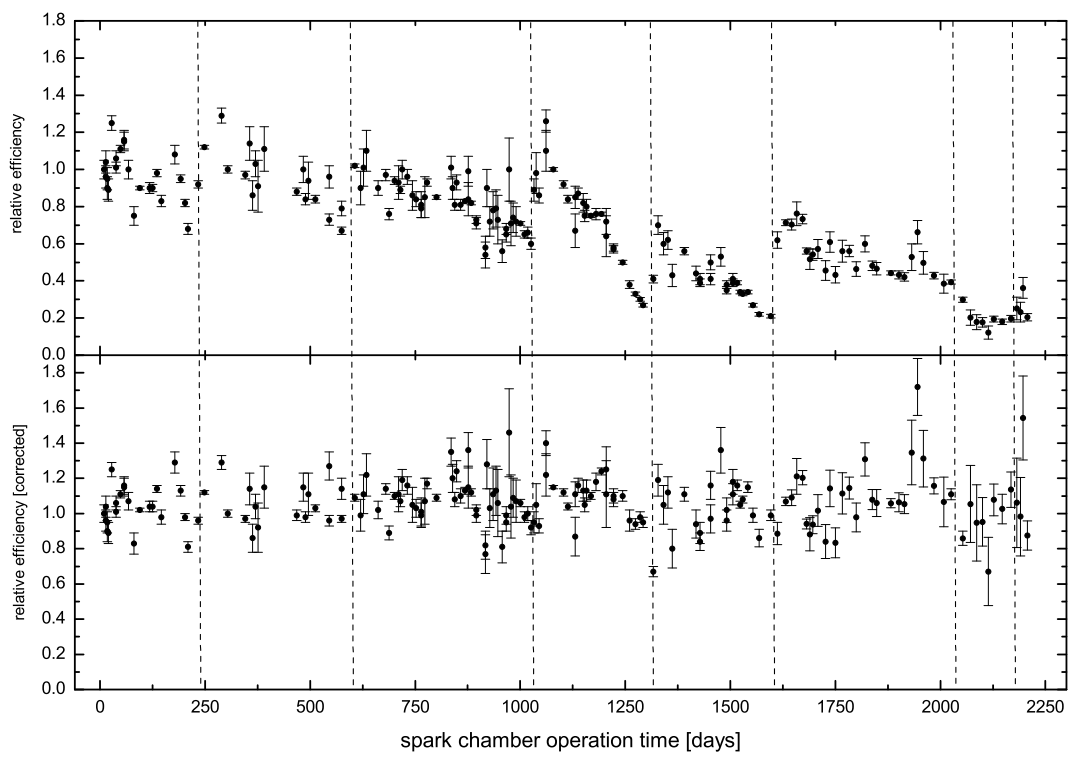

Figure 7 EGRET efficiency as function of observation time, Upper panel: before normalization, lower panel: after normalization has been applied.

So far, variablity studies have been performed largely on the basis of entries in EGRET source catalogs. The first systematic study by McLaughlin et al. 1996 made use of individual viewing periods from the 2EG catalog (Thompson et al. 1995), and has been expanded online to include CGRO cycle 3 observations. It has been used also by Wallace et al. 2000 to study short-term time variability on the basis of sub-viewing periods. The method is effectively a measure of inconsistency of the gamma-ray data with the assumption of a constant source flux (for details see McLaughlin et al. 1996).

A different approach to properly quantify flux variability has been carried out by Tompkins 1999, consitently computed for the 3EG catalog sources and source fluxes. A method has been introduced to obtain a fractional variability measure by calculating the likelihood for obtaining any source flux and compare to the actual observation (for details see Tompkins 1999).

Following a method successfully applied to radio data (Romero, Combi \& Colomb 1994), Zhang et al. 2000 and Torres et al. 2000 determined a gamma-ray flux variability measure by means of a weighted fluctuation index with pulsars as "standard candle". Unfortunately, both teams use different subsets of gamma-ray sources in the variability study which introduces problems to directly compare the results of the different meth- 
ods.

Although being a selection of steady sources by comparing detection significances at different observational timescales only (individual and combined viewing periods), Gehrels et al. 2000 effectively obtained a qualitative measure of source variabilty. This source ensemble is selected against one-time flaring sources (transients) and variable but dim sources close to the detection threshold of the EGRET instrument.

Each method attempted to distinguish gamma-ray sources by means of quantifying the degree of flux variability. At present, different classes of gamma-ray emitters are most clearly distinguishable in terms of variabilty by applying the method developed by Tompkins. Figure 8 gives the sketch of the separation achieved on the basis of the variabilty criterion $\tau$ (as the inverse fraction of the average and the standard deviation). Among the unidentified sources, there is a tendency that sources at low Galactic latitudes are less variable than at high Galactic latitudes. It is striking that the variability distribution of unidentified sources as a whole is distincly different than the one of pulsars and active galactic nuclei.

When comparing the results of the different methods against each other, the level of consistency among the results of the variability studies is incredibly low. Unfortunately, each study has been performed using different source ensembles, hampering efforts to trace the origin of such discrepancies. Figure 9 compares, for instance, results from the variability study by Tompkins with the one by McLaughlin, using sources common to two samples. Note that the difference of the number of viewing periods included in the study by Tompkins and by McLaughlin could not account for the spread in the variability results.

In consequence, not only the flux uncertainty of a particular source in a given time interval needs to be accounted for, also the uncertainties of the normalization procedure. Studies aimed to compute a common measure of gamma-ray flux variation should be aware of the level of underlying systematics before drawing conclusions. Variabilty examined up to the very detail for an individual source might stand here as an example, as recently revised for the high-latitude unidentified source 3EG J1835+5918 (Reimer et al. 2001). For many years believed to be a variable source of gamma-ray emission, only the combination of its expanded observational history, appropriate cuts to establish comparable quality in the data to be compared and an understanding of the systematics revealed that this source actually is compatible being a non-variable source, in this case with direct implications of its likely identification. 
12
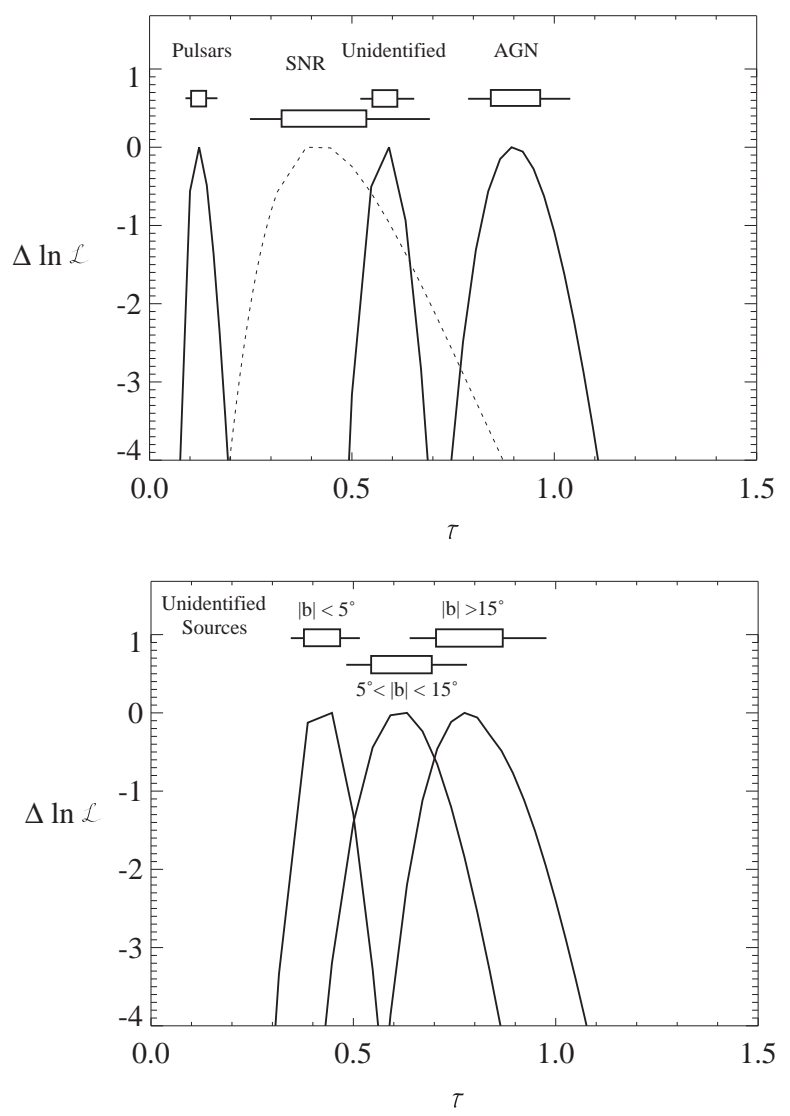

Figure 8 Variabilty index for different source populations. From: [Tompkins 1999]

\section{SPECTRAL CHARACTERISTICS OF GAMMA-RAY SOURCES}

The efficency of the EGRET spark chamber is not only a function of time, but also of the energy. Figure 10 shows the spark chamber efficiency as function of time for the ten energy intervals typically chosen for determining the spectrum of an EGRET detected gamma-ray source. For clarification, the normalization functions obtained by fitting the data (compare with upper panel Fig.7) are given here. Consequences beyond the systematics as already mentioned for gamma-ray source fluxes are expected.

The Third EGRET catalog lists for the majority of the sources the photon spectral index in $\mathrm{F}(\mathrm{E}) \sim \mathrm{E}^{-\gamma}$, consistently determined for the combined observations from CGRO observation cycles 1 to 4 . In var- 


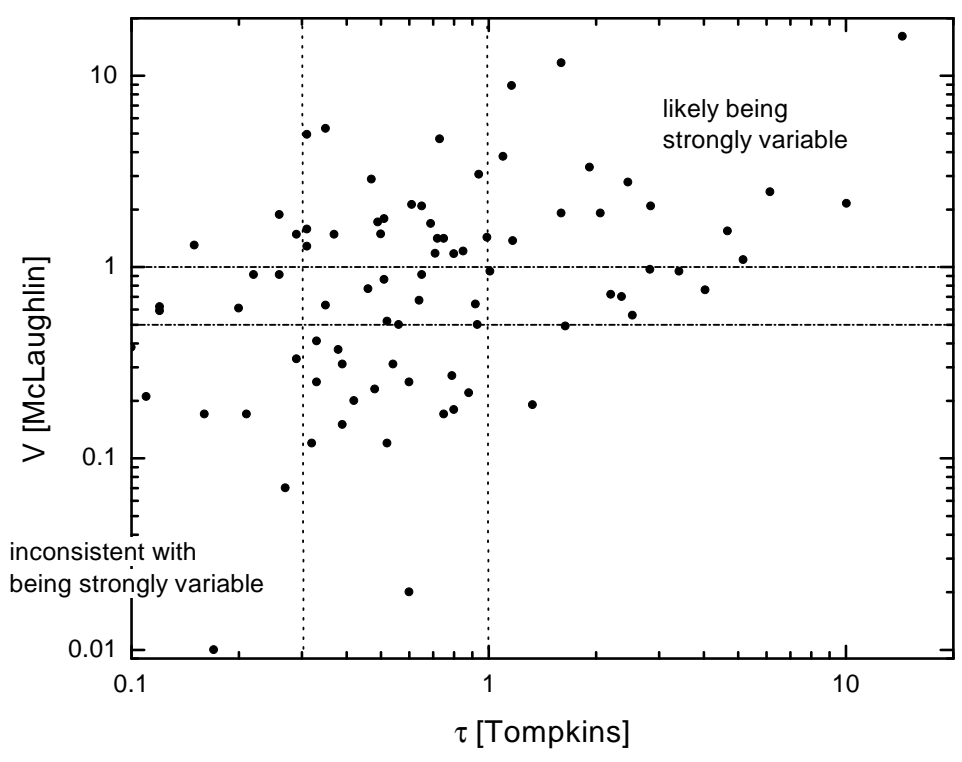

Figure 9 Comparison of the results from different variability studies, here given for McLaughlin et al. 1996 and Tompkins 1999. The vertical and horizontal grid represent the suggested boundaries by the autors for low variabilty, uncertain, and high variability characteristics.

ious cases the P1234 sum is not the most significant detection and a meaningful or even better spectrum could be determined in an individual viewing period. For the EGRET detected AGN a spectral study on the level of individual viewing periods has been performed (if sufficient counts have been recorded) by Mukherjee et al. 1997. Fierro et al. 1997 also published phase-resolved spectra for the brightest gamma-ray pulsars. At present, individual EGRET source spectra are investigated beyond a single power-law model fit (Bertsch et al. 2000). Additionally, the spectral index could be used in order to conclude on spectral variability. Such information goes beyond flux variability and an individual power-law spectral index only, but has not often be used in studies of individual as well as population studies so far.

The spectral characteristics of individual gamma-ray sources have been used in a similar way to attempt a distinction between source classes in population studies as variabilty, exclusivly on the basis of the photon spectral indices given in the 3EG catalog. Generally ignored by only picking up the numerical value of the spectral index when drawing conclusions, the significant uncertainties in the spectral index, especially for dim sources, put conclusions of distinct spectral characteristics rather into perspective. At present, conservative conclusions could only be 


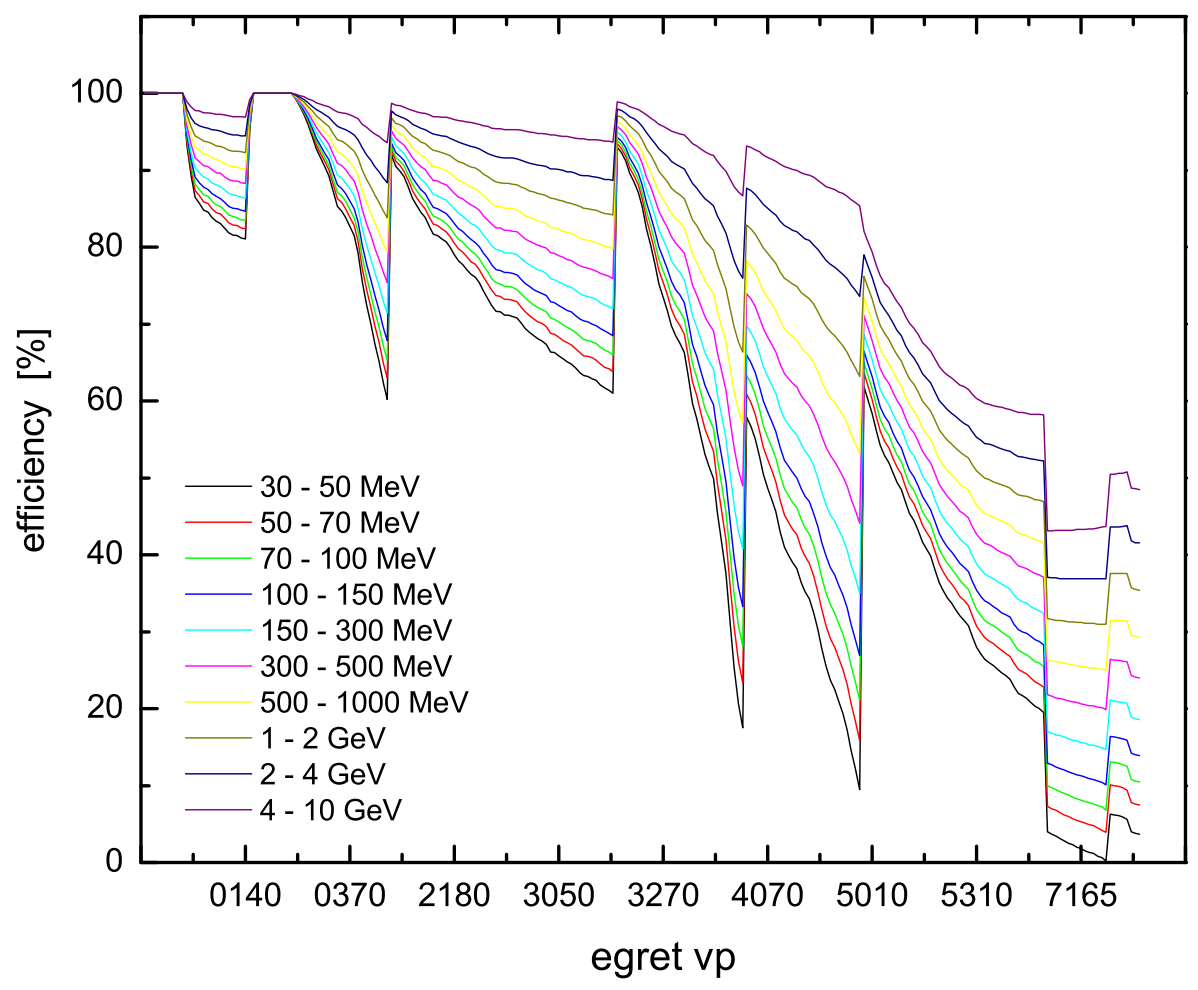

Figure 10 EGRET efficiency as function of observation time and energy. The ten subsequent energy bands are sketched which are used to determine source spectra. For clearer view, here the correction functions are plotted only.

drawn from the hardness of the spectrum of gamma-ray pulsars and the indication of a cut-off at GeV-energies. AGN seem to have a different spectrum when observed at high activity states (outbursts/flares) compared to their average spectra, however this needs to be investigated further. The vast majority of the unidentified EGRET sources are currently not distinguishable from the identified gamma-ray source populations by spectral characteristics only. Due to the wide spread found among the spectal index, and accounting for the uncertainties in the power-law spectral fits as well as the systematic bias towards finding hard spectrum gamma-ray sources significantly easier to discriminate against the diffuse emission component than soft spectra sources, it appears that viable conclusions might arise rather from the detailed spectrum of an individual source than from populations studies. Merck et al. 1996 conducted a survey for pulsar-like characteristics among the sources near the 

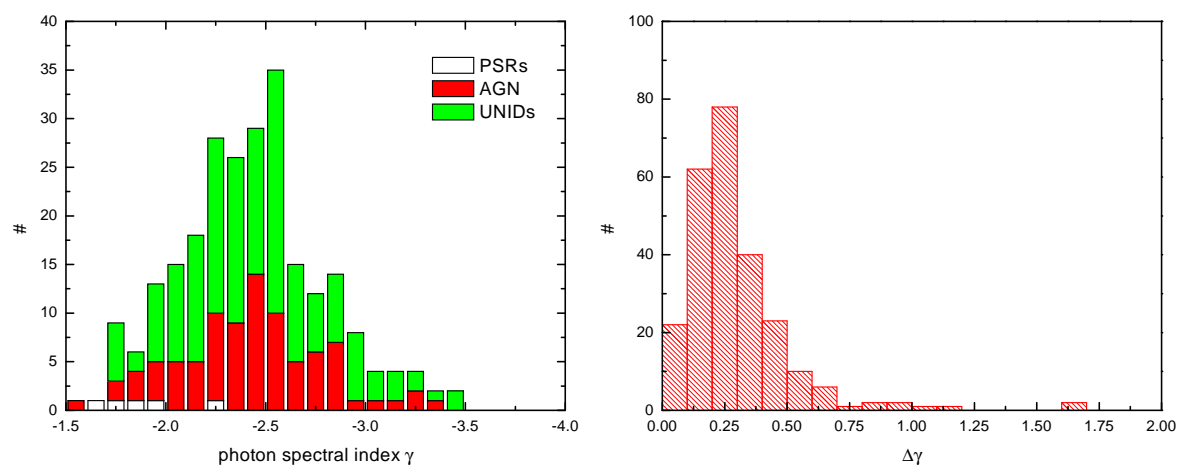

Figure 11 Distribution of the power-law spectral index for AGN, PSRs and unidentified EGRET sources and its uncertainty. It gives a vague impression about the difficulty to distinguish unidentified gamma-ray sources by its spectral characteristics only.

Galactic plane. At present, three of the sources suggested therein support the validity of this approach (PSR B1046-58/3EG J1048-5840, RX J2020.2+4026/3EG J2020+4017, SAX J0635+0533/3EG J0634+0521).

\section{SIGNATURES FROM SPATIAL, TEMPORAL AND SPECTRAL PROPERTIES}

Seeking signatures from the spatial, temporal and spectral characteristics of the unidentified EGRET sources is the subject of population studies. At present, the ensemble of persistent (Grenier 1999) or steady sources (Gehrels et al. 2000) appears to be the most promising in order to give hints on the nature of unidentified sources. Here I only sketch the general associations between spatial, temporal, and spectral properties for the unidentified EGRET sources and address the most prominent features or lack of features, respectively.

In Fig. 12a, the tendency to find hard spectrum sources predominantely close to the Galactic plane is apparent. However, this is at least partly due to the inability to discriminate soft spectrum sources near the plane against the dominant diffuse gamma-ray emission component. Fig. 12b gives the impression that variability is a common phenomenon for unidentified sources at all Galactic latitudes, although a clustering of more steady sources towards the Galactic plane is indicated, at least for the sources with extremely low probability to exhibit strong flux variability. Fig. 12c shows the correlation between spectral and variability 

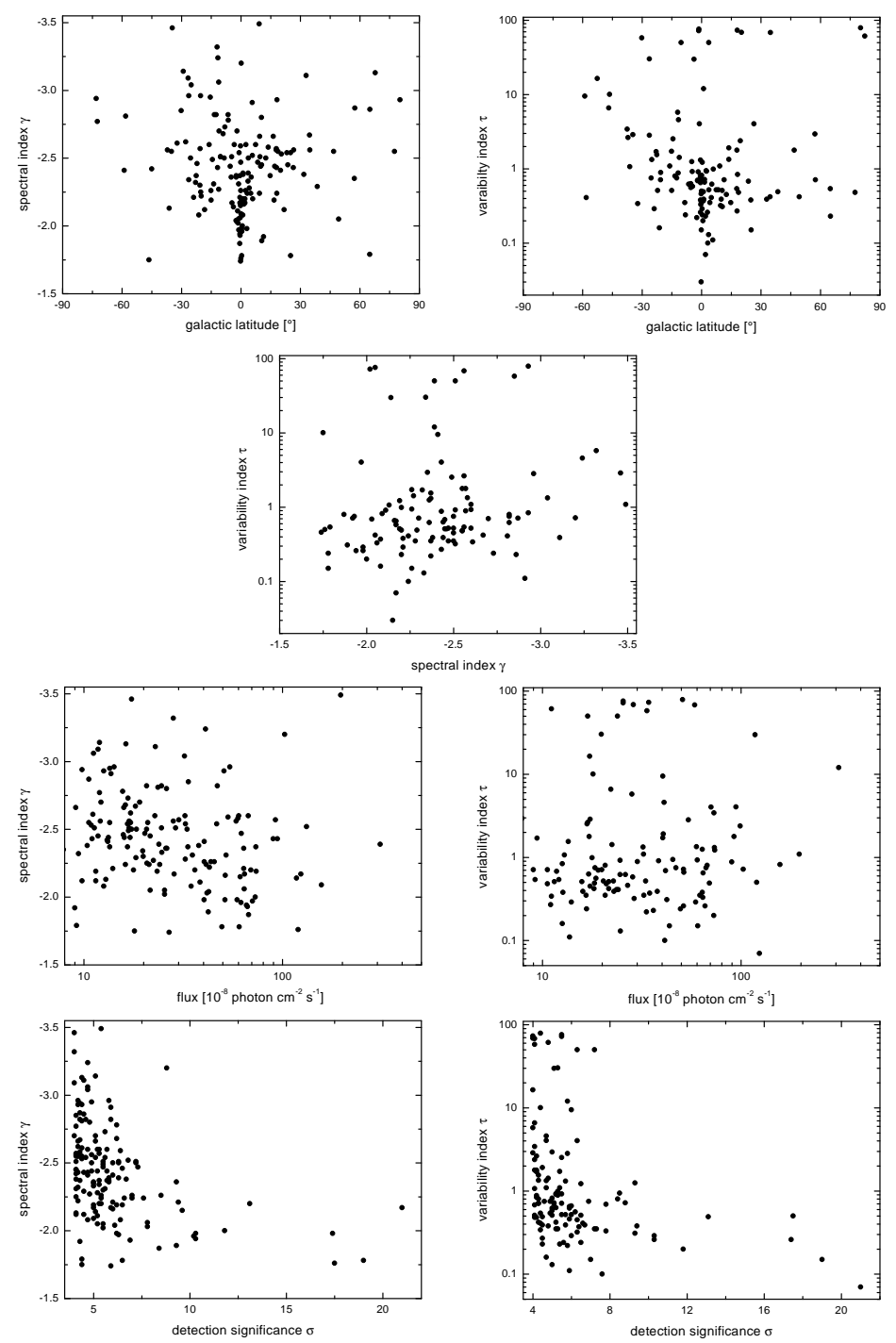

Figure 12 Associations between the observables determined to characterize the gamma-ray properties of unidentified EGRET sources. See text for details.

index. Only a rough tendency is indicated, that the softer sources are also characterized by higher variability. Counterexamples could be found for both extremes: hard spectrum sources exhibiting a high degree of flux variability and soft sources consistent with being nonvariable. Fig. 12d shows the flux distribution of unidentified sources. The few extremely high fluxes are the signatures of transient sources, arising from hard as 
well as soft spectrum emitters. Fig. 12e shows the same flux distribution against the variability index. The flaring or transient behaviour of unidentified sources is not in direct relation to either extrem flux values or strong source variability. This seems rather curious, but becomes obvious when comparing with a similar arrangement of these quantities for the EGRET detected AGN. Fig. $12 \mathrm{f}$ and $12 \mathrm{~g}$ show spectral and variability index as function of the source detection significance. High detection significances could be found predominantly for hard spectrum and low variable sources, giving the combination of both the rather distinct feature as apparent in the selections called "steady" (Gehrels et al. 2000) or "persistent" (Grenier et al. 1999).

However, nearly all signatures have to be put into perspective when supplemented by the appropriate uncertainties. The less apparent correlations are basically at the $1 \sigma$ level in the uncertainties of the observables themselves. Only carefully chosen combinations between the observables still reveal higher degrees of confidence in correlations among the observable parameter of the unidentified EGRET sources.

\section{CONCLUSIONS}

At present, we still fail to identify the nature of the majority of gamma-ray sources on the sky. However, the various observables at hand help to characterize individual sources rather well. For applications beyond individual sources (population studies, selected source ensembles) the degree of systematic biases and individual uncertainties needs to accounted for. Above all, in most cases gamma-ray astronomy still suffers from statistical limitations. Applying cuts is a valid procedure only if the particular cut is well understood in all its consequences for the data space. Conclusions drawn from subsets have to address its implication for the residual sources also. Generally, selections have to be made to avoid additional non-uniformities as already present in the $3 \mathrm{EG}$ source catalog. It needs to be investigated, at which level the known systematics and biases might put existing population studies in perspective. Efforts should be made to obtain more unbiased subsets instead of picking up any and each of the catalog listed gamma-ray sources. A compensation against the various biases by performing appropriate corrections is a requirement for comprehensive population studies.

The EGRET data will remain unique in gamma-ray astronomy until follow-up missions, in particular GLAST, will clarify a lot concerning the identity of individal gamma-ray sources and hypotheses of the composition of unidentified EGRET sources in the collective. Until then we have to work out the open questions on the basis of already acquired 
data. The tremendous potential offered by the nine years of EGRET data should not be neglected.

\section{Acknowledgments}

The author wish to thank Alberto Carramiñana and the staff of INAOE for the kind hospitality to make this workshop happened in a way as successfully and memorable as it apparently became. Also, I like to thank NRC for travel support.

\section{References}

Fierro, J.M. et al. 1997, ApJ 494, 734

Esposito, J.A. et al. 1999, ApJS 123, 203

Gehrels, N. et al. 2000, Nature 404, 363

Grenier, I., 1999, AIP Conf. Proc. 515, 261

Hartman, R.C. et al. 1999, ApJS 123, 79

Hunter, S.D. et al. 1997, ApJ 481, 205

Lamb, R.C. \& Macomb, D.J 1997, ApJ 488, 872

Mattox, J.R. et al. 1996, ApJ 461, 396

McLauchlin, M. et al. 1996, ApJ 473, 763

Merck, M. et al. 1996, A\&A Suppl. Ser. 120, 465

Mukherjee, R. et al. 1997, ApJ 490, 116

Özel, M.E. \& Thompson, D.J. 1996, ApJ 463, 105

Reimer, O. et al. 1997, Proc. 25th ICRC, Vol.3, 97

Reimer, O. et al. 2001, MNRAS, in press (astro-ph/0102150)

Romero, G.E. et al. 1994, A\&A 288, 731

Strong, A.W., Moskalenko, I.V. \& Reimer, O. 2000, ApJ 537, 763

Thompson, D.J. et al. 1995, ApJS 101, 259

Tompkins, W. 1999, PhD thesis Stanford University

Torres, D.F. et al. 2001, A\&A, in press (astro-ph/0007464)

Wallace, P. et al. 2000, ApJ 540, 184

Zhang, L., Zhang, Y.J. \& Cheng, K.S. 2000, A\&A 357, 957 